(C) 1996 Springer-Verlag New York Inc.

\title{
A Generalization of the Theory of Normal Forms
}

\author{
W. H. Warner, ${ }^{1}$ P. R. Sethna, ${ }^{\dagger, 1}$ and J. P. Sethna ${ }^{2}$ \\ 1 Aerospace Engineering and Mechanics, University of Minnesota, 107 Akerman Hall, 110 \\ Union St. S.E., Minneapolis, MN 55455, USA \\ 2 Department of Physics, Cornell University, Ithaca, NY 14853, USA
}

Received October 10, 1995; revised manuscript accepted for publication June 24, 1996 Communicated by Stephen Wiggins

\begin{abstract}
Summary. Normal forms allow the use of a restricted class of coordinate transformations (typically homogeneous polynomials) to put the bifurcations found in nonlinear dynamical systems into a few standard forms. We investigate here the consequences of relaxing the restrictions of the form of the coordinate transformations. In the Duffing equation, a logarithmic transformation can remove the nonlinearity: in one interpretation, the nonlinearity is replaced by a branch cut leading to a Poincaré section. When the linearized problem is autonomous with diagonal Jordan form, we can remove all nonlinearities order by order using these singular coordinate transformations.
\end{abstract}

Key words. normal forms, bifurcations, Duffing equation, Poincaré section, Lie transform, nonlinear Stokes phenomena

AMS Subject Classifications: 58F14, 58F36, 58F37

Normal form theory is a technique for transforming the ordinary differential equations describing nonlinear dynamical systems into certain standard forms. Using a particular class of coordinate transformations, one can remove the inessential part of higher-order nonlinearities. Unlike the closely related method of averaging, the standard development of normal form theory involves several technical assumptions about the allowed classes of coordinate transformations (often restricted to homogeneous polynomials). In a recent paper [1], the second author considered the equivalence of the methods of averaging and of normal forms. The references given there, particularly Chow and Hale [2], should be consulted for a full treatment of Lie transforms.

\footnotetext{
$\dagger$ Deceased, November 4, 1993.
} 
In this paper, we relax the restrictions on the transformations allowed. We start with the Duffing equation and show that a singular coordinate transformation can remove the nonlinearity associated with the usual normal form. We give two interpretations of this coordinate transformation, one with a branch cut reminiscent of a Poincare section. We then show that, when the generating problem is linear and autonomous with diagonal Jordan form, we can remove all nonlinearities order by order using singular coordinate transformations generated by the solution to the first-order linear partial differential equation produced by the Lie transform method of normal form theory. A companion paper [4] discusses these methods in a more general context and treats a specific example with a nondiagonal Jordan form for the generating matrix. The methods developed here are a specific and illuminating example of a recently developed "nonlinear Stokes phenomena" approach to bifurcation theory [5], [6].

\section{Duffing's Equation: Removing the Nonlinearities}

The second-order problems modeled by Duffing's equation or van der Pol's equation under near-resonance forcing are examples of the behavior that concerns us here. We use the notation of the Duffing example in [1, eqn. (37)]:

$$
\ddot{x}+\omega^{2} x+\varepsilon \tilde{c} \dot{x}+\varepsilon \tilde{h} x^{3}=\varepsilon \tilde{R} \cos (\Omega t)
$$

where $\Omega \approx \omega, \varepsilon$ is a small parameter, and the other constants are positive. If we manipulate the variables and parameters in a standard way (see [1]) by choosing

$$
\tilde{R}=\Omega^{2} R, \tilde{h}=\Omega^{2} h, \tilde{c}=\Omega c, \omega^{2}=\Omega^{2}(1+\varepsilon \sigma), \tau=\Omega t, y=d x / d \tau=x^{\prime}
$$

and changing to complex state variables

$$
p(\tau)=\frac{1}{2}(x+i y), \ldots, \bar{p}=\frac{1}{2}(x-i y),
$$

then eqn. (1) becomes

$$
p^{\prime}=-i p+i \varepsilon\left\{-\frac{1}{2}(\sigma-i c) p-\frac{1}{2}(\sigma+i c) \bar{p}-\frac{h}{2}(p+\bar{p})^{3}+\frac{R}{4}\left(e^{i \tau}+e^{-i \tau}\right)\right\}
$$

and its conjugate.

In [1], standard methods are used to show that the coordinate transformation

$$
p=z+\varepsilon W(z, \bar{z}, \tau), \ldots, \bar{p}=\bar{z}+\varepsilon \bar{W}(z, \bar{z}, \tau)
$$

with

$$
W=-\frac{1}{4}(\sigma+i c) \bar{z}+\frac{R}{8} e^{i \tau}+\frac{h}{4}\left(z^{3}-3 z \bar{z}^{2}-\frac{\bar{z}^{3}}{2}\right)
$$

can transform the $p$-equation into the normal form

$$
z^{\prime}=-i z+\varepsilon f_{1}(z, \bar{z}, \tau)+O\left(\varepsilon^{2}\right),
$$


where

$$
\begin{aligned}
f_{1} & =K_{1} z+K_{2} z^{2} \bar{z}+K_{3} e^{-i \tau}, \\
K_{1} & =-\frac{i}{2}(\sigma-i c), \quad K_{2}=-\frac{3 i h}{2}, \quad K_{3}=\frac{i R}{4} .
\end{aligned}
$$

It is the $z^{2} \bar{z}$ term that standard normal form theory considers as essential to keep at the cubic order for equations of this type.

Now consider the coordinate transformation from $(p, \bar{p})$ to $(u, \bar{u})$ given by

$$
\begin{aligned}
p & =u+\varepsilon V(u, \bar{u}, \tau) \\
& =u+\varepsilon\left[W(u, \bar{u}, \tau)+\frac{i}{2} f_{1}(u, \bar{u}, \tau) \ln \left(\frac{u}{\bar{u}}\right)\right]
\end{aligned}
$$

and its conjugate. Explicit computation shows that $u(\tau)$ satisfies a differential equation of the form

$$
u^{\prime}=-i u+O\left(\varepsilon^{2}\right) .
$$

By using a coordinate transformation with a logarithmic singularity at $u=0$, we have removed the first-order term in $\varepsilon$, including the $u^{2} \bar{u}$ term which normal form theory tells us is essential and irremovable.

A special case of equation (5) occurs when $f_{1}=z-z^{2} \bar{z}$ (note that this does not correspond to a Duffing equation with real coefficients). For this special case, the exact solution $z(\tau)=r(\tau) \exp (i \theta(\tau))$ to the nonlinear equation through order $\varepsilon$ terms can be written down for initial conditions $r(0)=r_{0} \neq 1, \theta(0)=\theta_{0}$ :

$$
r(\tau)=\frac{r_{0}}{\sqrt{r_{0}^{2}+\left(1-r_{0}^{2}\right) \exp [-2 \varepsilon \tau]}}, \quad \theta(\tau)=+\theta_{0}-\tau .
$$

Our transformation equation (7) generates the approximate solution

$$
r^{(1)}(\tau)=r_{0}+r_{0}\left(1-r_{0}^{2}\right)(\varepsilon \tau), \quad \theta^{(1)}=\theta(\tau)=\theta_{0}-\tau .
$$

Graphs of both types of functions starting from the same initial conditions $z(0)=(1+i) / 2$ are shown in Figures 1 and 2.

Figure 1 shows the trajectory corresponding to the exact solution $r(\tau)$ for $\varepsilon=0.05$.

Figure 2 shows the corresponding graph of the approximate solution $r^{(1)}(\tau)$. It is clear that the approximate solution spirals outward, crossing the limit cycle after a finite time $\tau_{0}$ of order $1 / \varepsilon$. One can verify that our approximate solution is the first term in the power series expansion of the exact solution in the parameter $\varepsilon$. Notice that the approximation is good to first order in $\varepsilon$, but not uniformly accurate in time.

Figure 3 shows trajectories for various initial conditions generated by our coordinate transformation $V$. Here we have restricted $V$ to a single Riemann sheet of the logarithm: hence the discontinuity at the branch cut along $\operatorname{Re}[z]<0, \operatorname{Im}[z]=0$. This branch cut is what allows the logarithm to "unwrap" the singularity of the Duffing equation. Thus it is natural not to continue increasing $\theta$ with time, but to restart the approximate solution every time one crosses the negative real axis. This may at first seem strange, as 


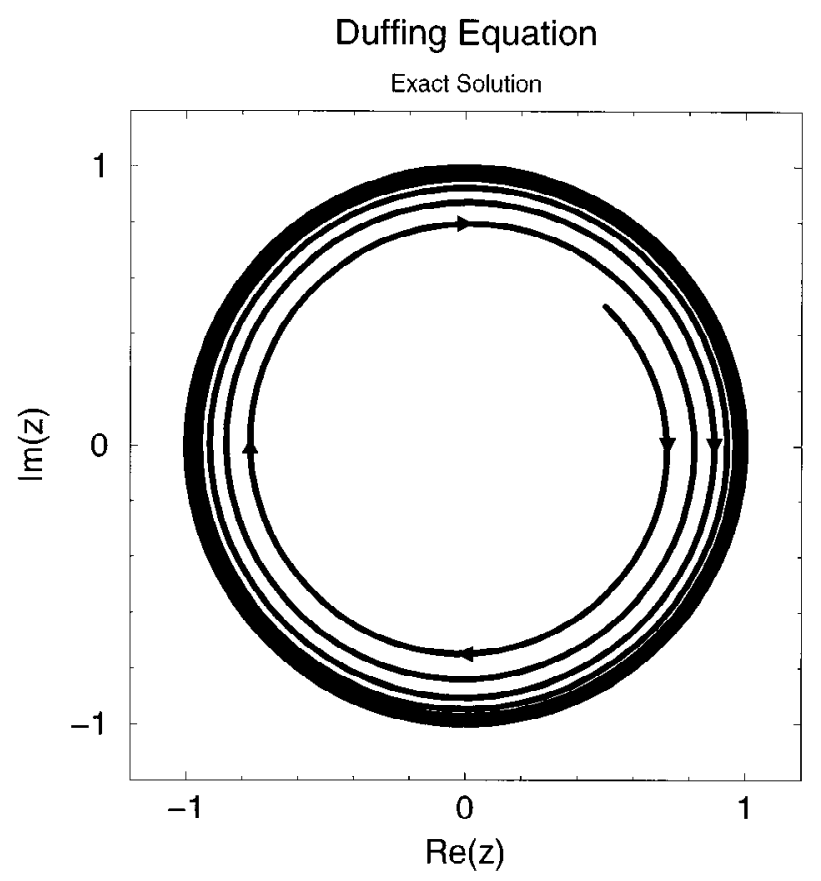

Fig. 1. Solution $r(\tau)$ for the Duffing equation, equation (9), with $\varepsilon=0.05$.

we are making a jump in the nonphysical variable $u(\tau)$. On the other hand, we are forced into this to make the coordinate transformation $V(u, \bar{u}, \tau)$ a single-valued function: the discontinuity in $u$ is needed to make the dynamics of $p=u+\epsilon V$ continuous. Our $O(\varepsilon)$ solution now systematically approximates a Poincaré first-return map $T$ along the negative real axis:

$$
T^{(1)}(r)=r+r\left(1-r^{2}\right)(2 \pi \varepsilon),
$$

giving a systematic approximation for the next intersection $T(r)$ of a curve which crosses $\operatorname{Im}[z]=0$ at $r=\operatorname{Re}(z)<0$. This interpretation of the dynamics preserves the qualitative behavior of the original dynamical system, although admittedly it does not produce an explicit analytical solution for the dynamics.

\section{Solving the Lie Differential Equation}

Why does normal form theory miss this useful transformation? (Alternatively, how does normal form theory avoid this nasty singular transformation?) We must look more closely at how the transformation of state variables is found by solving the partial differential equation produced by the Lie transform process.

For the case when the state equations have linear generating terms in diagonal Jordan form $\left(\mathbf{A}=\operatorname{diag}\left(\lambda_{\alpha}\right)\right.$ with no $\left.\lambda_{\alpha}=0, \alpha=1,2, \ldots, N\right)$, the state equation governing 


\section{Duffing to First Order}

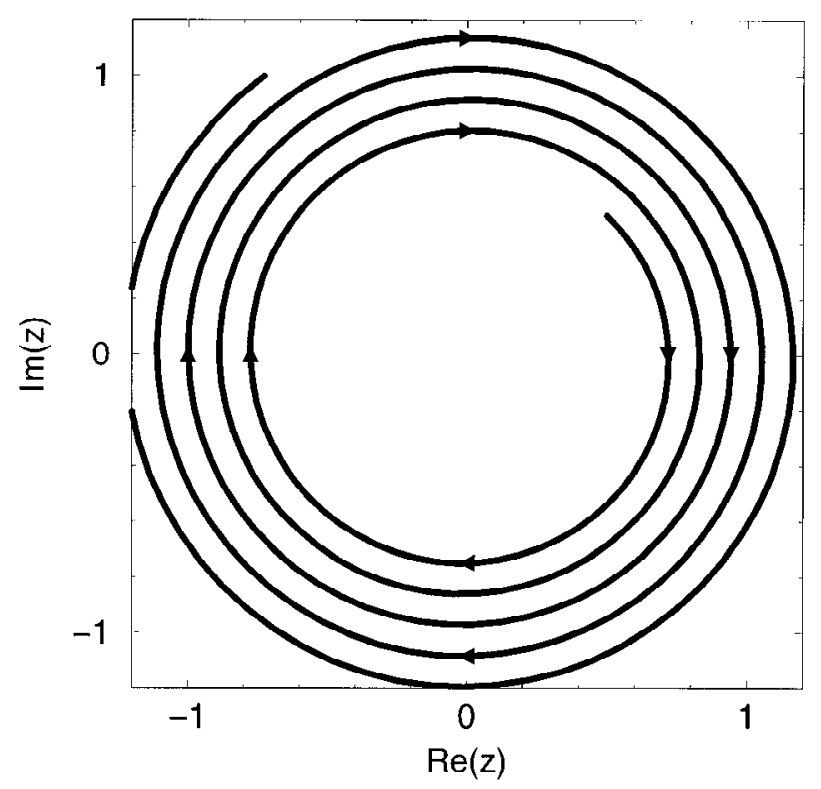

Fig. 2. Approximate solution $r^{(1)}(\tau)$ for the Duffing equation, equation (10), with $\varepsilon=0.05$.

the component $x_{\alpha}$ of $\mathbf{x}$ will be

$$
\frac{d x_{\alpha}}{d t}=\lambda_{\alpha} x_{\alpha}+\varepsilon f_{\alpha}^{(1)}(t, \mathbf{x})+\frac{\varepsilon^{2}}{2} f_{\alpha}^{(2)}(t, \mathbf{x})+\cdots
$$

The equation for the corresponding component $W_{\alpha}^{(1)} \equiv W_{\alpha}(t, \mathbf{y})$ in the transformation $\mathbf{x}=\mathbf{y}+\varepsilon \mathbf{W}^{(1)}+\left(\varepsilon^{2} / 2\right) \mathbf{W}^{(2)}+\cdots$ is the Lie equation

$$
L\left(W_{\alpha}\right) \equiv \frac{\partial W_{\alpha}}{\partial t}+\sum_{\beta} \lambda_{\beta} y_{\beta} \frac{\partial W_{\alpha}}{\partial y_{\beta}}-\lambda_{\alpha} W_{\alpha}=f_{\alpha}^{(1)}(t, \mathbf{y}) .
$$

For autonomous $f^{(1)}$, the $\partial / \partial t$ term on the left is dropped from $L$. In that case, by restricting the class of coordinate transformations to homogeneous polynomials, normal form theory cannot remove any nonlinearities in $f^{(1)}$ that lie in the null space of the Lie operator. These nonlinear terms therefore comprise the "normal form": all other terms are removed by the coordinate transformation. But why should the null space of $L$ (the set of functions satisfying $L(W)=0$ ) have anything to do with finding a particular solution to the equation $L(W)=f$ ?

To understand this, consider a different linear operator, corresponding to solutions of the forced harmonic oscillator:

$$
H(x) \equiv \ddot{x}+x=f(t)
$$




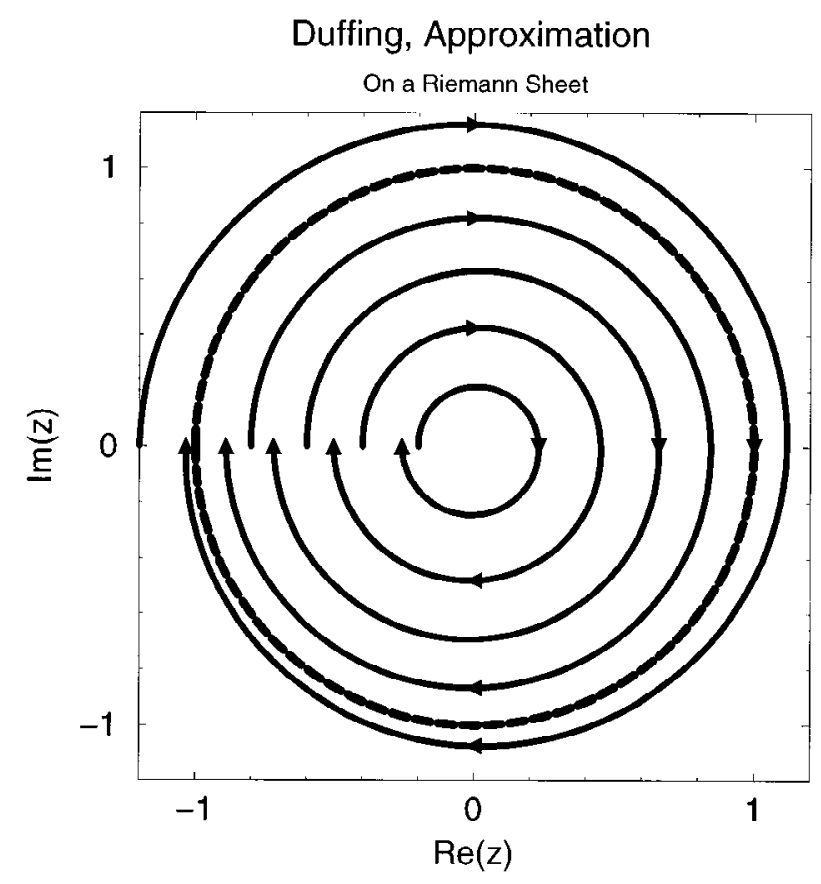

Fig. 3. Approximate solutions $r^{(1)}(\tau)$ (equation (10), $\varepsilon=0.05$ ) for various initial conditions, each confined to one sheet of the Riemann surface (beginning and ending along $\operatorname{Re}[z]<0$, $\operatorname{Im}[z]=0)$. The dashed line shows the limit cycle.

There is something special about solutions $x(t)$ for forcing functions in the null space of $H$. If we force at resonance $f(t)=\sin (t)$, then $f$ is in the null space of $H: H(f)=0$, and we see that the particular solution $x(t)=t \sin (t)$ is qualitatively different from the solution for $f(t)=\sin (\omega t)$ for other frequencies $\omega$. If we restrict the class of perturbations and solutions to finite sums of harmonic waves, then there would be no solution to $H(f)=\sin (t)$. Normal form theory makes precisely this kind of restriction: by restricting the perturbations and solutions to be homogeneous polynomials or in the Hamiltonian case to canonical transformations, they have defined away the possibly singular coordinate transformations that we study here.

We now demonstrate, for perturbations of the form $\mathbf{f}^{(1)}(t, \mathbf{y})$ above, that we can find solutions $W$ to the Lie operator partial differential equation

$$
L\left(W_{\alpha}\right) \equiv \frac{\partial W_{\alpha}}{\partial t}+\sum_{\beta} \lambda_{\beta} y_{\beta} \frac{\partial W_{\alpha}}{\partial y_{\beta}}-\lambda_{\alpha} W_{\alpha}=f_{\alpha}^{(1)}(t, \mathbf{y})
$$

in complete generality by reducing it to an ordinary one. Using the method of characteristics, one discovers (see Courant and Hilbert [3, p. 11] for a related transformation) 
that the coordinate transformation from $(\mathbf{y}, t)$ to $(\xi, \tau)$ given by

$$
\begin{aligned}
\xi_{1} & =y_{1}, \\
\xi_{\beta} & =\left(y_{\beta}^{1 / \lambda_{\beta}}\right) /\left(y_{1}^{1 / \lambda_{1}}\right), \quad \beta=2,3, \ldots, N, \\
\tau & =y_{1} \exp \left(-\lambda_{1} t\right)
\end{aligned}
$$

will reduce the partial differential equation to the ordinary differential equation

$$
\lambda_{1} \xi_{1} \frac{\partial V \alpha}{\partial \xi_{1}}=G_{\alpha}\left(\xi_{1}, \xi_{2}, \ldots, \xi_{n}, \tau\right)=\frac{1}{y_{\alpha}} f_{\alpha}^{(1)}(t, \mathbf{y})
$$

where the $V_{\alpha}=W_{\alpha} / y_{\alpha}$ are now to be considered as functions of $(\xi, \tau)$ and the $G$ 's are equal to the functions on the far right evaluated in the new variables. Our solution (7) removing the $O(\varepsilon)$ term in the Duffing equation was generated using precisely this method.

\section{Concluding Remarks}

We have studied the Duffing equation using a new approach to the calculation of an approximate solution. What about our methods in general? Where do they fit into the mathematical literature on bifurcations?

Our use of the method of characteristics is perfectly general: the same coordinate transformations used in the theory of normal forms can be shown [4] to remove all nonlinearities if the space of allowed functions is not restricted. Important questions remain about the nature of the higher order terms in $\varepsilon$ and about the estimates of the finite time for which the approximate solution is valid. (Here we find results valid to times of order $1 / \varepsilon$, but for the example of [4] times of order $1 / \varepsilon^{(1 / 4)}$ are found.)

Our simple interpretation of the resulting logarithmic transformation as a Poincare return map on coordinate patches has a well-developed context in the mathematical literature. For example, the study of singularities in complex analytic bifurcations [5] (equations of the form (5) but independent of $\bar{z}$ ) uses transformations defined on sectors which covered a punctured neighborhood of the origin-exactly like the overlapping patches we would use to unwrap the Riemann sheet of the logarithm. The corresponding work on real-analytic bifurcations [6] (their Andronov-Hopf family) analyzes the form and transformation properties of the first-return map and uses it to prove the existence of "linearizing charts." Our paper can be viewed as a less technical introduction to this mathematical method, emphasizing the calculational aspects derived from the alternative Lie transform approach to normal forms.

To summarize, one can take a dynamical system at a bifurcation and cast it into one of a small number of "normal forms" using a sequence of coordinate transformations restricted to homogeneous polynomials. We notice here that, abandoning this restriction, one can take a nonlinear bifurcation (the Duffing equation) and make it completely linear-with a coordinate transformation that has a branch cut through to the origin. The method naturally introduces the Poincaré first-return map as the transition function across the branch cut. 


\section{References}

[1] P. R. Sethna, On Averaged and Normal Form Equations, Nonlinear Dynamics, 7, 1-10 (1995).

[2] S. N. Chow \& J. R. Hale, Methods of Bifurcation Theory, Springer-Verlag, New York (1982).

[3] R. Courant \& D. Hilbert, Methods of Mathematical Physics, Vol. II: Partial Differential Equations, Wiley-Interscience Publishers, New York (1962).

[4] W. H. Warner. An Extension of Normal Form Methods for Calculating Approximate Solutions, Applied Mathematics: Methods and Applications (G. Oyibo, ed.), Proceedings of a conference in honor of Dr. George H. Handelman, Rensselaer Polytechnic Institute, Troy, NY, March 1995, Nova Science Publishers, Inc. (to appear).

[5] Yu. S. Il'yashenko, Nonlinear Stokes Phenomena, pp. 1-55, in Nonlinear Stokes Phenomena, Advances in Soviet Mathematics 14, edited by Yu. S. Il'yashenko, American Mathematical Society, Providence, RI (1991).

[6] Yu. S. Il'yashenko \& S. Yu. Yakovenko, Nonlinear Stokes Phenomena in Smooth Classification Problems, pp. 235-287, in Nonlinear Stokes Phenomena, Advances in Soviet Mathematics 14, edited by Yu. S. Il'yashenko, American Mathematical Society, Providence, RI (1991). 